\title{
Icosabutate, a Structurally Engineered Fatty Acid, Improves the Cardiovascular Risk Profile in Statin-Treated Patients with Residual Hypertriglyceridemia
}

\author{
John J.P. Kastelein ${ }^{a}$ Jonas Hallén ${ }^{b}$ Runar Vige ${ }^{b}$ David A. Fraser ${ }^{b}$ Rong Zhou ${ }^{c}$ \\ Svein Olaf Hustvedt ${ }^{b}$ David G. Orloffc Harold E. Bays ${ }^{d}$ \\ a Department of Vascular Medicine, Academic Medical Center, University of Amsterdam, Amsterdam,

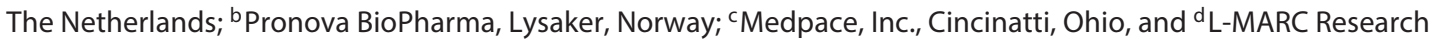 \\ Center, Louisville, Ky., USA
}

For editorial comment see p. 1

\section{Key Words}

Icosabutate - Triglycerides - Hypertriglyceridemia - Statins .

Low-density lipoprotein cholesterol · High-density

lipoprotein cholesterol · Apolipoprotein cholesterol III

\section{Abstract}

Objectives: To evaluate the efficacy and safety of icosabutate, an oral, once-daily, first-in-class medication, in reducing non-high-density lipoprotein cholesterol (non-HDL-C) in patients with persistent hypertriglyceridemia despite statin therapy. Methods: The study was designed to randomly assign 140 patients with fasting triglyceride levels $\geq 200$ but $<500 \mathrm{mg} / \mathrm{dl}$ on a stable dose of statin therapy to receive either masked icosabutate $600 \mathrm{mg}$ once daily or a control for 12 weeks. The primary end point was a percentage change in non-HDL-C from baseline to 12 weeks. Results: With icosabutate, non-HDL-C levels were reduced $(-9.2 \%)$ when compared with the control $(-0.4 \%)$ for a between-group difference of $-7.4 \%(p=0.02)$. Compared with the control, icosabutate reduced triglycerides $(-27.0 \%, p<0.001)$, verylow-density lipoprotein (VLDL) cholesterol $(-31.5 \%, p<$ $0.001)$ and apolipoprotein C-III $(-22.5 \%, \mathrm{p}<0.001)$. LDL-C levels did not change $(0.5 \%, p=0.87)$. HDL-C $(10.2 \%, p<$
0.001) was increased. After 113 subjects had been randomized, the study was terminated due to a partial clinical hold imposed by US regulators after observing QT prolongation at supratherapeutic doses of icosabutate in a dog study. In this study, adverse events were balanced between treatment arms, and there were no discontinuations due to adverse events. Conclusions: Icosabutate was efficacious in lowering non-HDL-C and other biomarkers of cardiovascular risk and was generally well tolerated.

(c) 2016 The Author(s)

Published by S. Karger AG, Basel

\section{Introduction}

Very-low-density lipoproteins (VLDL) and their remnants are triglyceride-rich particles that contain cholesterol and contribute to the overall atherogenic burden. Under hypertriglyceridemic conditions, the amount of cholesterol carried by the triglyceride-rich lipoproteins can be substantial. Elevations in triglycerides and triglyceride-rich lipoproteins are associated with an increased risk of atherosclerotic cardiovascular $(\mathrm{CV})$ events even in

David G. Orloff has deceased.

\section{KARGER}

E-Mail karger@karger.com www.karger.com/crd
(C) 2016 The Author(s)

Published by S. Karger AG, Basel 0008-6312/16/1351-0003\$39.50/0

This article is licensed under the Creative Commons AttributionNonCommercial-NoDerivatives 4.0 International License (CC BY NC-ND) (http://www.karger.com/Services/OpenAccessLicense) Usage and distribution for commercial purposes as well as any distribution of modified material requires written permission. 
Fig. 1. Study design.

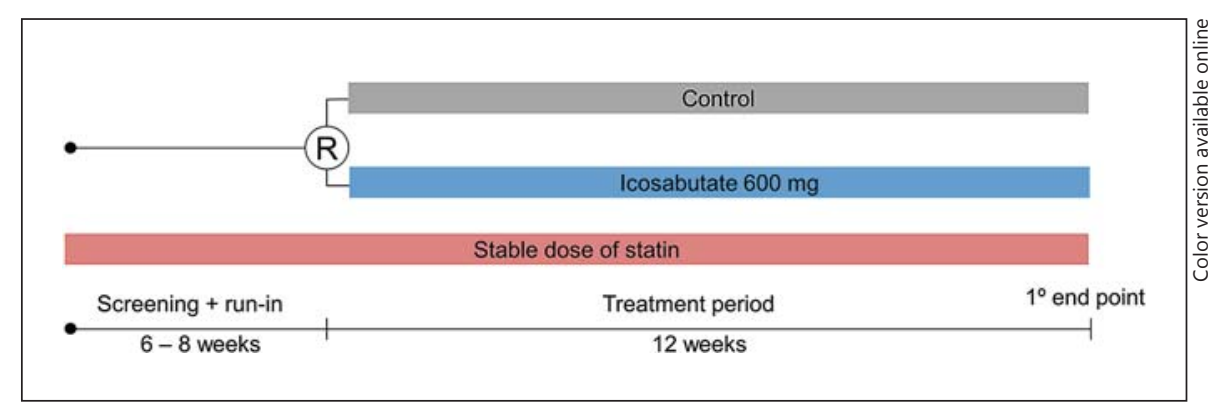

patients where well-controlled levels of low-density lipoprotein cholesterol (LDL-C) have been achieved by highintensity statin regimens [1-4]. Residual risk in hypertriglyceridemic patients is thus often addressed clinically by triglyceride-lowering therapies [5], even though this therapeutic approach remains disputed because randomized trials have lacked appropriately defined patient populations and failed to demonstrate definitive clinical outcomes benefit $[6,7]$. However, a growing body of evidence spanning observational, experimental and genetic studies as well as post hoc analyses from randomized trials supports the concept that triglyceride-related pathways are causally linked to the development of atherosclerosis [8-12]. Modulation of triglyceride metabolism thus still carries promise for addressing residual risk in patients with hypertriglyceridemia.

Icosabutate is an oral, once-daily, first-in-class, synthetically modified eicosapentanoic acid (EPA) molecule in clinical development. The structural modification of icosabutate minimizes esterification and thus limits both fatty acid activation and incorporation into complex lipids (unpublished data on file). The resulting high intracellular concentration of the free fatty acid form of icosabutate has potent effects upon lipid metabolism likely mediated through several independent mechanisms. In rodent dyslipidemia models, icosabutate treatment lowers the levels of both hepatic and plasma triglyceride and cholesterol [13]. Hepatic LDL receptor expression is increased along with markedly increased hepatic clearance of both plasma triglyceride and cholesterol [13]. In addition, hepatic apolipoprotein C-III (apo C-III) mRNA expression and plasma apo C-III concentration are reduced (unpublished data on file). These findings suggest potentially unique pleiotropic effects upon hepatic lipid metabolism. Icosabutate has been evaluated clinically in hypercholesterolemia and severe hypertriglyceridemia (triglycerides $>500 \mathrm{mg} / \mathrm{dl}$ ) $[14,15]$. In both these populations, icosabutate lowered triglycerides, apo C-III and non high-density lipoprotein cholesterol (non-HDL-C) whereas LDL-C responses were dependent on the baseline LDL-C and triglyceride values.

The primary objective of this clinical proof-of-concept study was to evaluate the efficacy, tolerability and safety of icosabutate $600 \mathrm{mg}$ once daily versus a control in reducing the totality of circulating atherogenic cholesterol, measured as non-HDL-C, and other lipid markers in patients with modest but persistent hypertriglyceridemia despite stable statin therapy.

\section{Materials and Methods}

Study Design and Organization

This was a randomized, double-blind, controlled study conducted at 40 sites in the USA. The study comprised a 4- to 6-week diet, lifestyle and medication stabilization/ washout period, a 2 -week lipid qualifying period and a 12 -week randomized, doubleblind treatment period (fig. 1). At the screening visit, all subjects received counseling regarding the National Cholesterol Education Program Therapeutic Lifestyle Changes Diet. The duration of the stabilization period was 4 weeks for subjects who were not on nonstatin lipid-altering therapy and 6 weeks for patients who were required to discontinue other lipid-altering therapies not allowed in the study (e.g. omega-3, fibrates and niacin).

The study was conducted according to Good Clinical Practice guidelines, the Declaration of Helsinki (2000) and the US 21 Code of Federal Regulations. The appropriate national and institutional regulatory and ethical boards approved the protocol and the informed consent document prior to initiation of the study. Participants provided signed informed consent before any study procedure was initiated. The trial was designed by the Sponsor (Pronova BioPharma) and Medpace Inc.; Medpace coordinated, monitored and provided site and data management. Recruitment began in November 2013 and the study was terminated in July 2014.

\section{Study Participants}

Inclusion study criteria included men and nonpregnant women between 18 and 79 years of age on a stable dose of statin therapy and with a fasting non-HDL-C level $>130 \mathrm{mg} / \mathrm{dl}$ and fasting triglyceride level $\geq 200$ and $\leq 500 \mathrm{mg} / \mathrm{dl}$ based on the average (arithmetic mean) of visits 1 and 2 in the lipid qualification period. If a subject's average non-HDL-C and triglyceride level fell outside the required range for entry into the study, an additional fasting lipid profile could be made 1 week later. If a $3 \mathrm{rd}$ sample was col- 
lected, entry into the study was to be based on the values from the last 2 visits.

Subjects had to be on a stable dose of statin (with or without ezetimibe) $\geq 4$ weeks prior to visit 2 (week 2 ). The same statin at the same dose was to be continued until the end of the study. The subjects on nonstatin lipid-lowering therapy had to be able to safely discontinue these at screening. Subjects were required to agree to maintain a stable diet, smoking habits and physical activity level throughout the study.

Exclusion criteria included patients with diagnosed lipoprotein lipase impairment or deficiency (Fredrickson type I), apo C-II deficiency or familial dysbetalipoproteinemia (Fredrickson type III), acute or chronic pancreatitis, symptomatic gallstone disease (unless previously treated with cholecystectomy), diagnosed hereditary or acquired myopathy, cancer in the last 5 years (basal or squamous-cell skin carcinoma was allowed), type 1 diabetes, uncontrolled type 2 diabetes [glycosylated hemoglobin $\left(\mathrm{HbA}_{1 \mathrm{c}}\right)>9.5 \%$ ], $\mathrm{BMI}>40$, previous bariatric surgery, an elevated QTc interval at screening, any ocular disorder for which topical ocular therapy was in use or chronically prescribed, nephrotic range proteinuria, a requirement for peritoneal dialysis or hemodialysis, uncontrolled hypertension, clinical evidence of hypothyroidism or thyroid hormone therapy that was not stable for $\geq 6$ weeks prior to screening, a positive test for HIV, hepatitis B or hepatitis C, a history or evidence of major and clinical significant disease that could have interfered with the conduct of the study and any CV event (e.g. stroke, myocardial infarction, life-threatening arrhythmia and coronary revascularization) within 6 months prior to study enrollment.

Exclusionary laboratory results included thyroid-stimulating hormone $>2.0 \times$ upper limit of normal (ULN), alanine aminotransferase or aspartate aminotransferase $>2.5 \times$ ULN (unless exercise-related), unexplained creatine kinase (CK) concentration $>5 \times$ ULN or CK elevation due to known muscle disease. Exclusionary medications included weight management pharmacotherapies known to affect intestinal fat absorption within 4 weeks of screening, insulin, chronic prescription pharmacotherapy for metabolic or $\mathrm{CV}$ disease management or risk factor modification (e.g. antihypertensive and antidiabetes medications) that were not stable for $\geq 4$ weeks prior to screening, HIV-protease inhibitors, cyclophosphamide or isotretinoin, tamoxifen, estrogens, progestins or topical testosterone that had not been stable for $\geq 4$ weeks prior to screening and the use of systemic corticosteroids at screening or planned use during the study.

\section{Study Drug, Randomization and Masking}

Subjects were randomly assigned in a 1:1 ratio to $600 \mathrm{mg}$ icosabutate (Pronova BioPharma Norge AS/BASF) or a matching control [Mygliol $812^{\circledR}$ (medium chain fatty acid)] at treatment initiation. Icosabutate and the control were administered orally as 6 capsules $(100 \mathrm{mg})$ once daily. Subjects were instructed to take their medication at approximately the same time each day (i.e. morning, midday or evening). On days when the subjects were scheduled for study site visits, the dose of study drug was to be administered by the site personnel following the collection of all fasting blood samples. Randomization was stratified by the use of the Medpace ClinTrak $^{\circledR}$ Interactive Voice Response System (CTIVRS). Subjects and investigators were masked to the treatment assignment, and subjects received a blinded study drug which was packaged and numbered as a kit for each individual based on the randomization scheme. The investigator or designee contacted CTIVRS in order to randomize the subject and to obtain the appropriate doubleblind kit identification. The subject randomization number assigned by CTIVRS corresponded to the number on the kit. The study drug was dispensed in amounts exceeding the amount required for the period of time until the next visit. Subjects were instructed to return all unused drugs at the next visit. Compliance to the study drug regimen was evaluated by counting the unused capsules.

\section{Study End Points}

The primary efficacy variable was a non-HDL-C percentage change from baseline to the end of the study (week 12). The week 12 end point was the average of the visit 8 (week 11) and visit 9 (week 12) measurements. Secondary end points included triglycerides, total cholesterol, HDL-C, LDL-C, VLDL-C, remnant-like protein (RLP) cholesterol, apo C-III, apo B, apo A1, lipoprotein a [Lp(a)], lipoprotein-associated phospholipase $\mathrm{A}_{2}$ (Lp-PLA $\mathrm{A}_{2}$, highsensitivity C-reactive protein (hsCRP), the EPA and docosahexanoic acid (DHA) content in the red blood cell membrane as well as metabolic variables such as $\mathrm{HbA}_{1 c}$, insulin resistance indices and the fasting plasma insulin and glucose values.

\section{Laboratory Efficacy and Safety Assessments}

All laboratory measurements were performed by Medpace Reference Laboratories (Cincinnati, Ohio, USA). Efficacy measurements included the serum concentrations of lipids including triglycerides, total cholesterol, VLDL-C, HDL-C, LDL-C and calculated non-HDL-C (total cholesterol - HDL-C), apo A1, apo B and apo C-III, Lp(a), Lp-PLA 2 , RLP cholesterol, hsCRP, EPA and DHA content, fasting plasma glucose, fasting plasma insulin and $\mathrm{HbA}_{1 \mathrm{c}}$.

LDL-C was measured via preparative ultracentrifugation (PUC) followed by analysis of the density $>1.006$ fraction on the Beckman Coulter AU Series Chemistry Analyzer. VLDL-C was calculated by subtracting the PUC-derived density $>1.006$ fraction produced from the total cholesterol value. Fasting plasma glucose, total cholesterol and triglycerides were all measured on this analyzer. The HDL-C analyses were performed by dextran sulfate precipitation followed by analysis of the supernatant on the same analyzer. Apo B, Apo Al and hsCRP were measured using rate nephelometry on a Siemens BNII. Apo C-III and RLP cholesterol were measured on the Randox Daytona Analyzer. Lp(a) was measured using an immunoturbidometric assay on the Beckman Coulter AU Series Chemistry Analyzer using an isoform-independent method. Lp-PLA 2 analysis was performed by the Atherotech Diagnostic Laboratory using the DYNEX-DSX TM Automated ELISA System. $\mathrm{HbA}_{1 \mathrm{c}}$ was measured on the Tosoh G7/G8 automated highperformance liquid chromatography analyzer. Plasma insulin was measured on Roche Immuno-Analyzers. Red blood cell fatty acid composition was analyzed by gas chromatography with flame ionization detection. Gas chromatography was carried out using a GC2010 gas chromatograph (Shimadzu Corp., Columbia, Md., USA) equipped with an SP2560, 100-m fused silica capillary column $(0.25$-mm internal diameter and 0.2-um film thickness; Supelco, Bellefonte, Pa., USA).

Laboratory safety assessments included serum chemistry, hematology and urinalysis. A physical examination was performed at screening, randomization and end of treatment. An off-site ophthalmologic examination was performed after eligibility was confirmed, prior to randomization and at week 11 or the end of the 
treatment by a licensed optometrist or ophthalmologist. The ophthalmologic investigation was included due to an observation of corneal pitting in a 4-week toxicology study on rats, even though this finding could not be replicated in other studies on rats or other species. Details regarding the ophthalmological assessment are included in the online supplementary material (for all online suppl. material, see www.karger.com/doi/10.1159/000445047). Vital signs (heart rate and blood pressure) as well as weight and waist circumference were also measured at every visit. A 12-lead ECG was performed following the screening period, at week 2 and at the end of treatment. Adverse events (AE) were assessed at visit 2 following the screening period and throughout the treatment period.

\section{Early Termination}

On 16 June 2014, the Sponsor received notification from the Food and Drug Administration (FDA) that all ongoing studies with icosabutate would be subject to limitations on duration and exposure until a thorough QT study had been conducted and the results assessed by the FDA. This decision was due to a signal of a QT prolongation in a chronic toxicology study conducted on dogs receiving a supratherapeutic dose of icosabutate. Based on the available phase I pharmacokinetic data, it was apparent that a minority of the subjects enrolled in our study would have attained a maximum concentration above the exposure limit set by the FDA. Recruitment was stopped on 18 June 2014. Dosing was discontinued on 20 June 2014 when all enrolled patients were contacted and told to discontinue the study drug and a final follow-up assessment was also scheduled. The study was terminated on 14 July 2014.

\section{Statistical Analyses}

The original protocol-defined primary analysis population for the efficacy end point was the intention-to-treat (ITT) population. Due to the early termination of the study, the statistical analysis plan was amended while the data were still blinded to only include patients who had a 12-week lipid measurement in the primary efficacy population. This change was introduced to ensure that potential late-onset effects of icosabutate on the primary and key secondary end points would also be captured in the primary efficacy analysis. All efficacy results would subsequently be confirmed in the ITT population which consisted of all randomized subjects who took at least 1 dose of investigational product, had a baseline efficacy measurement and at least 1 post-randomization efficacy measurement. This article reports efficacy results in the ITT population primarily and in the per-protocol population secondarily as a sensitivity analysis (online suppl. material). Results were consistent between the 2 analysis populations. Baseline and demographic characteristics are summarized for the randomized population which includes all subjects who signed the informed consent form and were assigned a randomization number. All safety analyses are based on all randomized subjects who received at least 1 dose of the study drug (safety population).

A sample size of 124 completed subjects was projected to provide $90 \%$ power to detect a difference of $10 \%$ between icosabutate and the control in percent change from baseline in fasting nonHDL-C levels, assuming a standard deviation of $17 \%$ and a significance level of 0.05 . To accommodate the withdrawal of subjects from randomization to the completion of the double-blind treatment period, a total of 140 randomized subjects was planned. When the study was terminated early, 81 subjects had completed 12 weeks of treatment. With the same assumptions, this sample
Table 1. Demographic and baseline characteristics (randomized population)

\begin{tabular}{lcc}
\hline Characteristics & $\begin{array}{l}\text { Control group } \\
(\mathrm{n}=57)\end{array}$ & $\begin{array}{l}\text { Icosabutate } \\
\text { group } \\
(\mathrm{n}=56)\end{array}$ \\
\hline $\begin{array}{l}\text { Age, years } \\
\text { Male }\end{array}$ & $\begin{array}{c}58.0 \pm 10.4 \\
\text { Race }\end{array} \quad \begin{array}{c}58.7 \pm 9.6 \\
\quad \text { Asian }\end{array}$ & $31(55)$ \\
$\quad$ Black/African-American & $3(0)$ & $0(0)$ \\
$\quad$ White & $53(93)$ & $2(4)$ \\
$\quad$ Other & $1(2)$ & $52(93)$ \\
Ethnicity & $12(21)$ & $2(4)$ \\
$\quad$ Hispanic or Latino & $31.7 \pm 4.5$ & $11(20)$ \\
BMI & $14(25)$ & $19 \pm 3.9$ \\
Diabetes & $42(74)$ & $41(73)$ \\
Hypertension & & $12(21)$ \\
Intensity of statin therapy & $11(20)$ & $35(57)$ \\
$\quad$ Low potency & $35(63)$ & $12(21)$ \\
$\quad$ Medium potency & $9(16)$ & $0(0)$ \\
$\quad$ Higher potency & $1(2)$ & \\
Dose missing &
\end{tabular}

Values are expressed as $\mathrm{n}(\%)$ or means \pm SD.

size provided approximately $75 \%$ power to detect a $10 \%$ difference in non-HDL-C between icosabutate and the control.

The primary efficacy analysis was performed using an analysis of covariance (ANCOVA) model with treatment, gender and the use of statin therapy at randomization as factors and the baseline triglyceride value as a covariate. For the primary analysis with parametric methods, the statistical modeling assumptions were examined. If significant departures from normality and/or homogeneity of variance were observed, a new analysis was performed on the overall relative ranks of the efficacy variable. As almost all data significantly departed from normality, nonparametric results are presented. Median values were generated by the Hodges-Lehmann statistic and Hodges-Lehmann asymptotic 95\% confidence intervals $(\mathrm{CI})$ were the primary test of statistical significance. Wilcoxon rank-sum $\mathrm{p}$ values were also generated as a secondary significance measure. No disagreement between Hodges-Lehmann and Wilcoxon rank-sum $\mathrm{p}$ values was observed. Statistical programming and analyses were performed with SAS ${ }^{\circledR}$ v9.3.

\section{Results}

\section{Patient Disposition and Baseline Characteristics}

Of the 461 subjects screened, 113 were randomized into the study, i.e. 57 to the control and 56 to icosabutate (fig. 2). Thirty-two subjects discontinued the study; 27 had to be discontinued by the Sponsor due to the termination of the study. Of the remaining 5 subjects who 


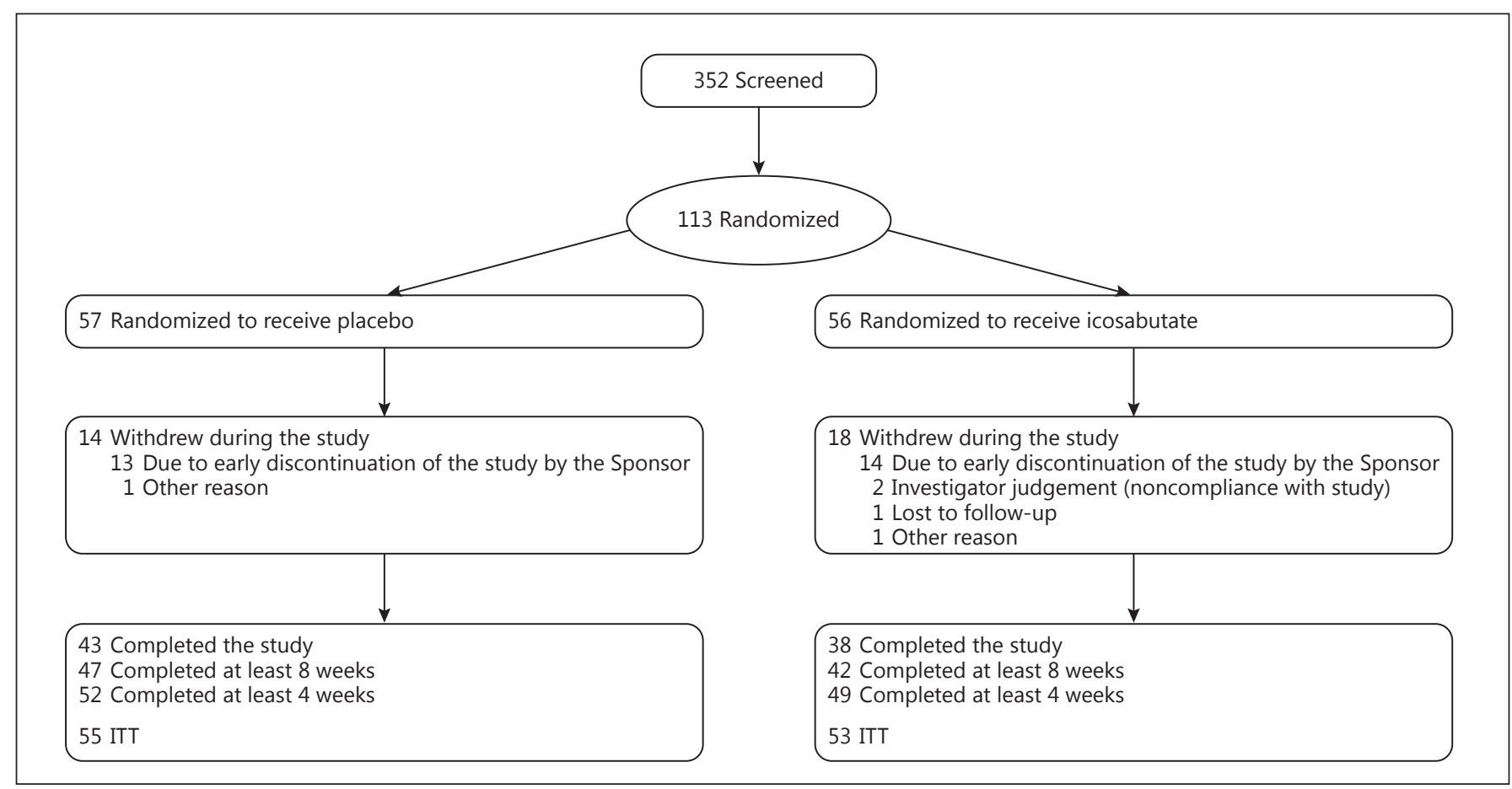

Fig. 2. Patient disposition.

Table 2. Baseline, end-of-treatment and median percent change values for the primary end point according to treatment group (ITT population)

\begin{tabular}{lcc}
\hline Non-HDL-C, mg/dl & Control $(\mathrm{n}=55)$ & Icosabutate $(\mathrm{n}=53)$ \\
\hline Baseline (Q1, Q3) & $165.5(147.0,173.0)$ & $162.5(144.5,178.0)$ \\
At 12 weeks (Q1, Q3) & $163.0(148.5,177.0)$ & $149.0(132.0,162.5)$ \\
\% change from baseline (Q1, Q3) & $-0.4(-11.2,10.1)$ & $-9.2(-18.1,3.5)$ \\
\% change vs. control (95\% CI) & & $-7.4(-13.5$ to -1.2$)$ \\
p value & & 0.020 \\
\hline
\end{tabular}

Q1 = First quartile; Q3 = third quartile.

discontinued, 4 were allocated to the icosabutate group, i.e. 2 were discontinued based on investigator judgement of noncompliance with the study protocol, 1 was lost to follow-up and 1 was discontinued for unspecified reasons.

Demographic and baseline characteristics of randomly assigned subjects are listed in table 1 . The mean $( \pm$ SD) age of the patients was $58 \pm 10$ years and the mean BMI was 31.6 ; $58 \%$ were men, $93 \%$ were white, $29 \%$ had diabetes and $74 \%$ had hypertension. The population was well-balanced with respect to all recorded characteristics (table 1). Mean percent compliance to study medication was $97 \%$ for the control group and $95 \%$ for the icosabutate group.

Icosabutate as an Add-On to Statins in Hypertriglyceridemia

\section{Efficacy End Points}

The median change (\%) in non-HDL-C was $-9.2 \%$ for the icosabutate group and $-0.4 \%$ for the control group, for an adjusted change of $-7.4 \%(p=0.02$; table 2$)$. Compared with baseline and with the control, icosabutate significantly reduced triglycerides $(-42.2$ and $-27.0 \%, \mathrm{p}<$ $0.001)$, VLDL-C $(-39.8$ and $-31.5 \%, \mathrm{p}<0.001)$, apo C-III $(-35.1$ and $-22.5 \%, \mathrm{p}<0.001)$, apo $\mathrm{B}(-5.9$ and $-7.8 \%$, $\mathrm{p}=0.024)$, the apo $\mathrm{B} /$ apo $\mathrm{A} 1$ ratio $(-0.05$ and $-0.08, \mathrm{p}=$ $0.014)$ and significantly increased HDL-C (9.1 and $10.2 \%$, $\mathrm{p}<0.001$ ) (table 3; fig. 3). Compared with baseline and with the control, icosabutate numerically reduced RLP cholesterol $(-26.8$ and $-13.4 \%, p=0.068)$, while there was no statistically significant or clinically meaningful change 
Table 3. Baseline, end-of-treatment and median percent change values for secondary lipid, apolipoprotein and inflammatory marker end points according to treatment group (ITT population)

\begin{tabular}{|c|c|c|c|c|c|c|c|}
\hline \multirow{2}{*}{$\begin{array}{l}\text { Variable, } \\
\mathrm{mg} / \mathrm{dl}\end{array}$} & \multirow[t]{2}{*}{ Treatment } & \multirow[t]{2}{*}{$\mathrm{n}$} & \multirow{2}{*}{$\begin{array}{l}\text { Baseline median } \\
(\mathrm{Q} 1, \mathrm{Q} 3)\end{array}$} & \multirow{2}{*}{$\begin{array}{l}\text { Week } 12 \text { median } \\
(\mathrm{Q} 1, \mathrm{Q} 3)\end{array}$} & \multirow{2}{*}{$\begin{array}{l}\text { Change (\%) from } \\
\text { baseline median } \\
(\mathrm{Q} 1, \mathrm{Q} 3)\end{array}$} & \multicolumn{2}{|l|}{ Icosabutate vs. control group } \\
\hline & & & & & & $\begin{array}{l}\text { median \% change from } \\
\text { baseline }(95 \% \mathrm{CI})^{\mathrm{b}}\end{array}$ & $\begin{array}{l}\text { Wilcoxon } \\
\text { rank-sum } \\
\mathrm{p} \text { value }\end{array}$ \\
\hline \multirow[t]{2}{*}{ Triglycerides } & Control & 55 & $255.5(213.0,369.5)$ & $236.0(202.0,317.0)$ & $-11.1(-22.4,4.9)$ & $-27.0(-34.5$ to -19.1$)$ & $<0.001$ \\
\hline & Icosabutate & 53 & $269.5(222.0,319.5)$ & $156.0(136.0,207.0)$ & $-42.2(-46.7,-24.7)$ & & \\
\hline \multirow[t]{2}{*}{ VLDL-C } & Control & 54 & $41.0(34.0,69.0)$ & $41.5(31.0,59.0)$ & $-5.5(-25.3,17.8)$ & $-31.5(-44.4$ to -19.2$)$ & $<0.001$ \\
\hline & Icosabutate & 50 & $42.5(31.0,56.0)$ & $26.3(19.0,35.0)$ & $-39.8(-57.4,-11.6)$ & & \\
\hline \multirow[t]{2}{*}{ HDL-C } & Control & 55 & $43.0(34.5,47.5)$ & $41.0(36.0,47.0)$ & $-1.1(-7.7,5.0)$ & $10.2(5.7$ to 15.2$)$ & $<0.001$ \\
\hline & Icosabutate & 53 & $45.0(37.0,48.0)$ & $47.0(40.0,54.5)$ & $9.1(0.0,17.7)$ & & \\
\hline \multirow{2}{*}{$\begin{array}{l}\text { Total } \\
\text { cholesterol }\end{array}$} & Control & 55 & $200.5(190.0,219.0)$ & $203.5(185.5,222.5)$ & $-1.5(-8.1,6.4)$ & $-3.4(-8.3$ to 1.8$)$ & 0.174 \\
\hline & Icosabutate & 53 & $206.0(187.5,223.5)$ & $197.0(179.0,212.0)$ & $-4.3(-13.0,3.6)$ & & \\
\hline \multirow[t]{2}{*}{ LDL-C } & Control & 54 & $115.0(94.0,136.0)$ & $115.3(99.5,130.0)$ & $0.1(-12.2,13.2)$ & $0.5(-7.5$ to 9.0$)$ & 0.871 \\
\hline & Icosabutate & 50 & $123.0(90.0,135.0)$ & $119.5(103.5,132.5)$ & $-1.1(-14.6,17.2)$ & & \\
\hline \multirow{2}{*}{$\begin{array}{l}\text { RLP } \\
\text { cholesterol }\end{array}$} & Placebo & 47 & $16.0(12.0,22.0)$ & $13.0(9.0,18.0)$ & $-12.0(-40.0,13.6)$ & $-13.4(-28.2$ to 0.9$)$ & 0.068 \\
\hline & Icosabutate & 42 & $15.5(12.0,18.0)$ & $10.0(9.0,14.0)$ & $-26.8(-43.8,-11.1)$ & & \\
\hline \multirow[t]{2}{*}{ Apo A1 } & Placebo & 55 & $145.0(134.0,161.0)$ & $143.0(131.0,163.0)$ & $-2.0(-6.6,9.8)$ & $2.1(-1.7$ to 6.3$)$ & 0.288 \\
\hline & Icosabutate & 53 & $152.0(134.0,168.0)$ & $154.0(142.0,171.0)$ & $-0.6(-3.3,11.1)$ & & \\
\hline \multirow[t]{2}{*}{ Apo B } & Control & 55 & $107.0(100.0,119.0)$ & $112.0(96.0,126.0)$ & $4.3(-9.2,11.5)$ & $-7.8(-14.1$ to -1.1$)$ & 0.024 \\
\hline & Icosabutate & 53 & $110.0(101.0,127.0)$ & $102.0(92.0,117.0)$ & $-5.9(-14.0,4.8)$ & & \\
\hline \multirow[t]{2}{*}{ Apo C-III } & Control & 47 & $17.3(13.6,20.6)$ & $14.9(12.2,18.2)$ & $-11.0(-25.1,0.5)$ & $-22.5(-29.5$ to -14.2$)$ & $<0.001$ \\
\hline & Icosabutate & 42 & $17.1(13.7,19.5)$ & $11.3(9.0,13.2)$ & $-35.1(-43.2,-22.7)$ & & \\
\hline \multirow{2}{*}{$\begin{array}{l}\text { Apo } \mathrm{B} / \mathrm{A} 1 \\
\text { ratio }\end{array}$} & Placebo & 55 & $0.770(0.660,0.870)$ & $0.760(0.670,0.880)$ & $0.010^{\mathrm{a}}(-0.080,0.120)$ & $-0.080(-0.130$ to -0.010$)$ & 0.014 \\
\hline & Icosabutate & 53 & $0.740(0.660,0.840)$ & $0.640(0.580,0.820)$ & $-0.050^{\mathrm{a}}(-0.190,0.050)$ & & \\
\hline \multirow[t]{2}{*}{$\operatorname{Lp}(\mathrm{a})$} & Placebo & 47 & $24.0(7.0,105.0)$ & $25.0(8.0,95.0)$ & $0.0(-6.8,21.1)$ & $14.3(0.0$ to 28.6$)$ & 0.056 \\
\hline & Icosabutate & 42 & $28.5(7.0,105.0)$ & $30.5(15.0,135.0)$ & $21.6(0.0,50.0)$ & & \\
\hline \multirow[t]{2}{*}{ hsCRP } & Placebo & 47 & $2.70(1.50,5.00)$ & $3.20(1.50,5.90)$ & $0.00(-1.30,1.30)^{\mathrm{a}}$ & $-0.80(-1.80$ to -0.00$)$ & 0.031 \\
\hline & Icosabutate & 42 & $2.20(0.90,5.40)$ & $1.70(0.60,3.90)$ & $-0.40(-2.20,0.00)^{\mathrm{a}}$ & & \\
\hline \multirow[t]{2}{*}{$\mathrm{Lp}-\mathrm{PLA}_{2}$} & Placebo & 47 & $214.4(191.0,236.7)$ & $205.6(173.2,235.0)$ & $-8.1(-15.3,3.6)$ & $1.6(-4.6$ to 7.0$)$ & 0.590 \\
\hline & Icosabutate & 42 & $197.4(170.7,226.7)$ & $188.6(167.3,204,6)$ & $-6.0(-15.4,3.7)$ & & \\
\hline
\end{tabular}

$\mathrm{n}=$ Number of patients; $\mathrm{Q} 1$ = first quartile; $\mathrm{Q} 3$ = third quartile.

${ }^{\text {a }}$ Absolute change. ${ }^{\mathrm{b}}$ Hodges-Lehmann asymptotic $95 \%$ CI.

in LDL-C (-1.1 and 0.5\%, p = 0.87) and apo A1 ( -0.6 and $2.1 \%, \mathrm{p}=0.29$; table 3$)$. Overall, the observed changes in lipids were similar between the ITT population and the 12-week per-protocol population (see online suppl. material). Changes in apo B were directionally consistent between the 2 populations, but only the ITT analysis indicated statistical significance.

For inflammatory markers, icosabutate significantly reduced CRP versus the baseline and controlvalues $(-0.40$ and $-0.80 \mathrm{mg} / \mathrm{dl}, \mathrm{p}=0.031$ ), but Lp-PLA ${ }_{2}$ was not changed (table 3). Compared to the control, icosabutate significantly increased plasma EPA by $52 \%(\mathrm{p}<0.001)$ and significantly reduced plasma arachidonic acid by $7 \%$ ( $<<$
$0.001)$. There was no change in plasma DHA $(-6.2 \%, \mathrm{p}=$ $0.09)$. Fasting plasma glucose, $\mathrm{HbA}_{1 c}$, fasting plasma insulin and the homeostatic model assessment of insulin resistance did not significantly change (see online suppl. material).

\section{Safety End Points}

During the study, 65 (58\%) of the subjects, i.e. 31 (55\%) in the control group and $34(61 \%)$ in the icosabutate group, had at least 1 treatment-emergent AE (TEAE; table 4). No subjects died during the study. Two subjects in the control group and no subjects in the icosabutate group had serious AEs. Most TEAEs during the study were mild 
Table 4. Safety and tolerability by treatment group

\begin{tabular}{llll}
\hline & $\begin{array}{l}\text { Control } \\
(\mathrm{n}=56)\end{array}$ & $\begin{array}{l}\text { Icosabutate } \\
(\mathrm{n}=56)\end{array}$ & $\begin{array}{l}\text { Total } \\
(\mathrm{n}=112)\end{array}$ \\
\hline AE & & & \\
Subjects with any AE & $31(55.4)$ & $34(60.7)$ & $65(58.0)$ \\
Subjects with any TEAE & $27(48.2)$ & $29(51.8)$ & $56(50.0)$ \\
Maximum severity of TEAE & & & $36(32.1)$ \\
$\quad$ Mild & $17(30.4)$ & $19(33.9)$ & $19(17.0)$ \\
$\quad$ Moderate & $9(16.1)$ & $10(17.9)$ & $1(0.9)$ \\
$\quad$ Severe & $1(1.8)$ & $0(0.0)$ & $22(19.6)$ \\
Subjects with any TEAE considered related to the study drug & $5(8.9)$ & $17(30.4)$ & $2(1.8)$ \\
Subjects with any serious AE & $2(3.6)$ & $0(0.0)$ & $1(0.9)$ \\
Subjects with any serious TEAE & $1(1.8)$ & $0(0.0)$ & $0(0.0)$ \\
Subjects with any TEAE leading to discontinuation or withdrawal & $0(0.0)$ & $0(0.0)$ & $17(15.2)$ \\
\hline TEAEs occurring in $>5 \%$ of subjects in any treatment group & & & $6(5.4)$ \\
Gastrointestinal disorders & $3(5.4)$ & $14(25.0)$ & $6(5.4)$ \\
$\quad$ Diarrhea & $1(1.8)$ & $5(8.9)$ & $22(19.6)$ \\
$\quad$ Nausea & $0(0.0)$ & $5(8.9)$ & $6(5.4)$ \\
Infections and infestations & $12(21.4)$ & $10(17.9)$ & $10(8.9)$ \\
General disorders & $3(5.4)$ & $3(5.4)$ & $6(5.4)$ \\
Muscle and connective tissue & $4(7.1)$ & $6(10.7)$ & $9(8.0)$ \\
Injury, poisoning and procedural complications & $6(10.7)$ & $0(0.0)$ & $7(6.3)$ \\
Investigations & $3(5.4)$ & $6(10.7)$ & $5(4.5)$ \\
Eye disorders & $5(8.9)$ & $2(3.6)$ & $3(5.4)$ \\
Skin & $2(3.6)$ & & \\
\hline$\quad$ Values are expressed as n $\%)$. & & & \\
\hline
\end{tabular}

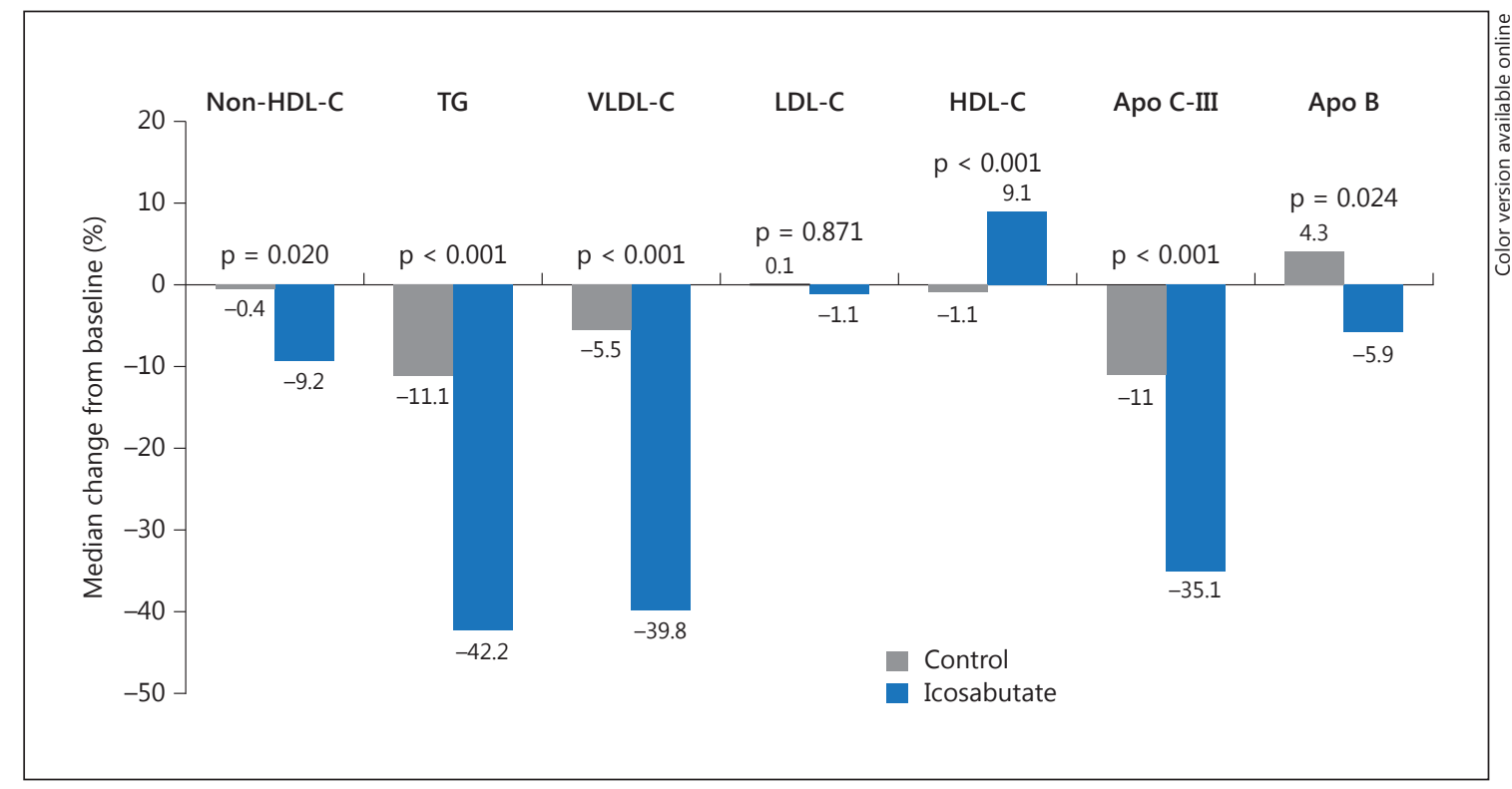

Fig. 3. Primary and key secondary efficacy end points. Bars represent the median change from baseline to the end of treatment. Median values were generated by the Hodges-Lehmann statistic, and p values (Wilcoxon rank-sum) are for differences between the icosabutate group and the control group. TG = Triglycerides. 
Table 5. Summary of electrocardiogram assessment by treatment group

\begin{tabular}{lll}
\hline Assessment & $\begin{array}{l}\text { Control } \\
\text { group }\end{array}$ & $\begin{array}{l}\text { Icosabutate } \\
\text { group }\end{array}$ \\
\hline At baseline screening & $(\mathrm{n}=56)$ & $(\mathrm{n}=56)$ \\
Normal & $31(55.4)$ & $31(55.4)$ \\
Abnormal, not clinically significant & $25(44.6)$ & $25(44.6)$ \\
Abnormal, clinically significant & $0(0)$ & $0(0)$ \\
At week 12/end of treatment & $(\mathrm{n}=56)$ & $(\mathrm{n}=54)$ \\
Normal & $32(57.1)$ & $31(57.4)$ \\
Abnormal, not clinically significant & $24(42.9)$ & $23(42.6)$ \\
Abnormal, clinically significant & $0(0)$ & $0(0)$ \\
\hline
\end{tabular}

Values are expressed as n (\%).

or moderate in severity; 1 subject (control) had a severe TEAE. There were no TEAEs that led to study drug discontinuation. The most common TEAEs belonged to system organ class infections and infestations and gastrointestinal disorders. No ECGs were considered to have any clinically relevant abnormal findings by the investigators (table 5).

Two cardiac-related AEs were reported in the icosabutate arm. One was sinus bradycardia, classified as mild in severity and which resolved. The other event was a mild QT prolongation reported immediately after investigators were informed by the Sponsor that the study was being discontinued due to the finding of a QT prolongation in an animal toxicology study. The event was classified as mild and it resolved. The corresponding ECG was not classified by the investigator to have any clinically relevant abnormalities (table 5).

There were no signs of any treatment-related ocular side effects at the off-site ophthalmological assessment. In total, there were 5 eye-related TEAEs, 4 in the control group and 1 in the icosabutate group. No clinically meaningful adverse changes in safety laboratory variables, ECG variables, vital signs or physical examination findings were noted.

\section{Discussion}

In this randomized, proof-of-concept trial, we studied the ability of icosabutate $600 \mathrm{mg}$ once daily to lower nonHDL-C versus a control in patients with persistent hypertriglyceridemia on a stable dose of a statin. We found that icosabutate significantly lowered the levels of non-HDL-
$\mathrm{C}$, triglycerides, triglyceride-rich lipoprotein cholesterol and apo C-III and significantly increased HDL-C, but LDL-C levels were unchanged. The results support the hypothesis that icosabutate may offer a complementary therapeutic approach when added to statin therapy for the further lowering of atherogenic cholesterol and several other biomarkers of CV risk in subjects with hypertriglyceridemia. Icosabutate was generally well tolerated with no imbalances in total AEs between treatment groups, no discontinuations due to AEs and no serious AEs in the icosabutate group.

After 113 patients had been randomized, the trial was terminated by the Sponsor when a maximum exposure limitation was imposed by the FDA due to observation of a modest QT interval prolongation at supratherapeutic doses in a 12-month study on dogs that was being run in parallel. In our study, no cardiac safety concerns emerged, but a thorough QT study is required to ultimately clarify any potential clinical implications of the observations made in the animal study. A thorough QT study on humans is planned and the protocol has been reviewed by the FDA.

Statin therapy can lower LDL-C by up to $50 \%$, but does not fully address lipid-driven risk in patients with persistent hypertriglyceridemia as reflected in increased nonHDL-C, increased triglyceride-rich lipoproteins and low HDL-C $[15,16]$. The significant non-HDL-C reduction conferred by icosabutate was entirely driven by a reduction in triglyceride-rich lipoprotein cholesterol and accompanied by an increase in HDL-C. Furthermore, apo C-III, a key upstream regulator of triglyceride metabolism, was reduced by $35 \%$. Taken together, these lipid shifts are consistent with a powerful modulation of triglyceride metabolism and the triglyceride-HDL axis and reflect the close and complex biological interdependence between triglycerides, cholesterol and their associated lipoproteins [17]. Several of the lipid variables affected by icosabutate are validated prospective biomarkers of $\mathrm{CV}$ risk and many have also been linked with adverse clinical outcomes in Mendelian randomization studies [2, 10, 1820]. Nevertheless, lipids remain surrogate markers of risk, and a CV outcomes study is needed to sufficiently define the full benefit-risk profile of icosabutate in patients with atherogenic dyslipidemia.

The most selective triglyceride-lowering drugs currently available are prescription omega- 3 fatty acids (OM3-FA) and fibrates. In the absence of direct head-tohead studies, potential similarities and differences between these drug classes and icosabutate must be interpreted cautiously. Triglyceride and non-HDL-C respons-
10

Cardiology 2016;135:3-12 DOI: $10.1159 / 000445047$
Kastelein/Hallén/Vige/Fraser/Zhou/ Hustvedt/Orloff/Bays 
es with $600 \mathrm{mg}$ icosabutate appear to be at least as large as that achieved by $4 \mathrm{~g}$ of OM3-FA or fenofibric acid in similar populations [21-23]. The magnitude of apo C-III reduction from baseline with icosabutate closely mirrors the findings in patients with severe hypertriglyceridemia, where icosabutate reduced apo C-III by $41 \%$ versus at baseline. In comparison, OM3-FA reduced apo C-III by $10-15 \%$ versus at baseline in similar populations [24,25].

Levels of LDL-C did not significantly change with icosabutate treatment. In patients with pronounced hypertriglyceridemia, interventions that lower triglyceride levels may raise LDL-C and HDL-C as atherogenic cholesterol shifts from the triglyceride-rich lipoprotein to the LDL and HDL particles. This remodeling is particularly marked in populations with triglyceride levels $>500 \mathrm{mg} /$ $\mathrm{dl}$ and has been observed with OM3-FA, fibrates, icosabutate and apo C-III antisense therapy [15, 26-28]. Although it is important to interpret changes in LDL-C levels in the context of changes in non-HDL-C and apo B, both of which provide a more complete picture of cholesterol-driven CV risk, an increase in LDL-C may complicate clinical management and pose significant regulatory hurdles. With the caveat that a noninferiority limit was not prespecified, the absence of any LDL-C increase with icosabutate despite the magnitude of the triglyceride reduction and the increase in HDL-C, is an important and potentially differentiating finding.

There were no serious AEs in the icosabutate-treated patients but there were 2 in the control group. Overall, the proportion of TEAEs was balanced between the control and icosabutate treatment groups. More subjects in the icosabutate arm reported gastrointestinal-related side effects than in the control arm. These events were predominantly mild in severity. An increased risk of gastrointestinal-related side effects has not been observed in previous studies of icosabutate. No changes were observed in fasting plasma glucose, $\mathrm{HbA}_{1 \mathrm{c}}$ or fasting plasma insulin. This is in contrast to a previous study on subjects with severe hypertriglyceridemia, where icosabutate significantly lowered fasting plasma insulin levels. It is relevant to note that subjects in the former study had, on average, higher baseline fasting insulin levels. In any case, the absence of any adverse impact on glucose metabolism differentiates icosabutate from other lipid-lowering therapies such as statins. Importantly, the addition of icosabutate to a stable statin regimen was not associated with any apparent increase in risk of either liver enzyme elevations or myopathy.

The results of our study should be viewed in the light of the following considerations. First, it was a small, proof-of-concept trial performed as part of a clinical development program. It was not designed to define the dose-response curve of icosabutate or to address questions of clinical benefit beyond the lipid and metabolic effects. Additional information on the clinical profile of icosabutate in patients with atherogenic dyslipidemia awaits larger studies of longer duration. Second, due to the early termination of the study, the primary analysis population was changed, following the last patient visit but prior to unblinding the database, from the ITT to the 12 -week completer population. Results were internally consistent in the primary analysis and ITT population. Third, the patient population enrolled in this study had relatively high LDL-C and non-HDL-C levels at baseline despite the fact that only $20 \%$ of them were on a high-dose statin regimen. This persistent hypercholesterolemia can partially be attributed to the inclusion criteria, but nevertheless suggests that a proportion of patients did not receive their maximally tolerated dose of statins.

In conclusion, we found that in subjects with persistent hypertriglyceridemia on stable doses of statin, icosabutate was efficacious and generally well-tolerated in lowering non-HDL-C and other lipid and lipoprotein biomarkers associated with residual risk of $\mathrm{CV}$ events.

\section{Acknowledgements}

We thank the Icosabutate Phase 2 Investigators for their contributions and Carrie Messerschmitt (Medpace Inc.) and Mette Hallén (Pronova BioPharma) for trial management.

\section{Conflict of Interest}

This trial was funded by Pronova BioPharma (Lysaker, Norway). Dr. Hallén, Mr. Vige, Dr. Faser and Dr. Hustvedt are employees of Pronova BioPharma. Dr. Bays and Prof. Kastelein have received research funding and/or consultancy honoraria from Pronova BioPharma. The following authors report other relationships with industry that might pose a potential conflict of interest: Dr. Bays (research grants: Amarin, Amgen, Ardea, Arisaph, Catabasis, Cymabay, Eisai, Elcelyx, Eli Lilly, Esperion, Hanmi, Hisun, Hoffman LaRoche, Home Access, Janssen, Johnson and Johnson, Merck, Necktar, Novartis, Novo Nordisk, Omthera, Orexigen, Pfizer, Pronova, Regeneron, Sanofi, Takeda, and TIMI. Consultancy/ speaker: Alnylam, Amarin, Amgen, Astra Zeneca, Eisai, Eli Lilly, Merck, Novartis, NovoNordisk, Regeneron, Sanofi and Takeda). Prof. Kastelein (Aegerion, Amgen, AstraZeneca, Boehringer Ingelheim, Catabasis, Cerenis, CSL Behring, Dezima Pharmaceuticals, Eli Lilly, Esperion, Isis, Merck, Novartis, Regeneron, Sanofi, The Medicines Company, UniQure, Cymabay, Gemphire). 


\section{References}

1 Nordestgaard BG, Varbo A: Triglycerides and cardiovascular disease. Lancet 2014;384:626635.

2 Miller M, Cannon CP, Murphy SA, et al: Impact of triglyceride levels beyond low-density lipoprotein cholesterol after acute coronary syndrome in the PROVE IT-TIMI 22 trial. J Am Coll Cardiol 2008;51:724-730.

3 Schwartz GG, Abt M, Bao W, et al: Fasting triglycerides predict recurrent ischemic events in patients with acute coronary syndrome treated with statins. J Am Coll Cardiol 2015;65:2267-2275.

4 Faergeman O, Holme I, Fayyad R, et al: Plasma triglycerides and cardiovascular events in the Treating to New Targets and Incremental Decrease in End-Points through Aggressive Lipid Lowering trials of statins in patients with coronary artery disease. Am J Cardiol 2009; 104:459-463.

5 Maki KC, Bays HE, Dicklin MR: Treatment options for the management of hypertriglyceridemia: strategies based on the best-available evidence. J Clin Lipidol 2012;6:413-426.

6 ACCORD Study Group: Effects of combination lipid therapy in type 2 diabetes mellitus. N Engl J Med 2010;362:1563-1574.

7 Keech A, et al, FIELD Study Investigators: Effects of long-term fenofibrate therapy on cardiovascular events in 9795 people with type 2 diabetes mellitus (the FIELD study): randomised controlled trial. Lancet 2005;366 1849-1861.

8 Scott R, O'Brien R, Fulcher G, et al: Effects of fenofibrate treatment on cardiovascular disease risk in 9,795 individuals with type 2 diabetes and various components of the metabolic syndrome: the Fenofibrate Intervention and Event Lowering in Diabetes (FIELD) study. Diabetes Care 2009;32:493-498.

9 Triglyceride Coronary Disease Genetics Consortium and Emerging Risk Factors Collaboration, Sarwar N, et al: Triglyceride-mediated pathways and coronary disease: collaborative analysis of 101 studies. Lancet 2010;375: 1634-1639.

10 Varbo A, Benn M, Tybjaerg-Hansen A, Jorgensen $A B$, Frikke-Schmidt R, Nordestgaard
BG: Remnant cholesterol as a causal risk factor for ischemic heart disease. J Am Coll Cardiol 2013;61:427-436.

11 Varbo A, Benn M, Nordestgaard BG: Remnant cholesterol as a cause of ischemic heart disease: evidence, definition, measurement, atherogenicity, high risk patients, and present and future treatment. Pharmacol Ther 2014 141:358-367.

12 Goldberg IJ, Eckel RH, McPherson R: Triglycerides and heart disease: still a hypothesis? Arterioscler Thromb Vasc Biol 2011;31:17161725.

13 Fraser DA, Skjaeret T, Qin Y, et al: Abstract 18507: icosabutate, a novel structurally enhanced fatty-acid increases hepatic uptake of cholesterol and triglycerides in conjunction with increased hepatic LDL receptor expression. Circulation 2014;130(suppl 2):A18507.

14 Qin Y, Hallén J, Skjæret T, et al: Abstract 11889: phase Ib study of icosabutate, a novel structurally enhanced fatty acid, in subjects with hypercholesterolemia. Circulation 2014; 130(suppl 2):A11889.

15 Bays HE, Hallén J, Vige R, et al: Icosabutate for the treatment of very high triglycerides: a placebo-controlled, randomized, doubleblind, 12-week clinical trial. J Clin Lipidol 2016;10:181-191.

16 Jones $\mathrm{PH}$, Davidson $\mathrm{MH}$, Stein EA, et al: Comparison of the efficacy and safety of rosuvastatin versus atorvastatin, simvastatin, and pravastatin across doses (STELLAR* Trial). Am J Cardiol 2003;92:152-160.

17 Szapary PO, Rader DJ: The triglyceride-highdensity lipoprotein axis: an important target of therapy? Am Heart J 2004;148:211-221.

18 Sharrett AR, Ballantyne CM, Coady SA, et al: Coronary heart disease prediction from lipoprotein cholesterol levels, triglycerides, lipoprotein(a), apolipoproteins A-I and B, and HDL density subfractions: the Atherosclerosis Risk in Communities (ARIC) Study. Circulation 2001;104:1108-1113.

19 TG and HDL Working Group of the Exome Sequencing Project, National Heart, Lung, and Blood Institute, Crosby J, et al: Loss-offunction mutations in APOC3, triglycerides, and coronary disease. N Engl J Med 2014;371: 22-31.

20 Jorgensen $A B$, Frikke-Schmidt R, Nordestgaard BG, Tybjaerg-Hansen A: Loss-of-function mutations in APOC3 and risk of ischemic vascular disease. N Engl J Med 2014;371: $32-41$.

21 Maki KC, Orloff DG, Nicholls SJ, et al: A highly bioavailable omega-3 free fatty acid formulation improves the cardiovascular risk profile in high-risk, statin-treated patients with residual hypertriglyceridemia (the ESPRIT trial) Clin Ther 2013;35:1400-1411 e1401-e1403.

22 Davidson MH, Stein EA, Bays HE, et al: Efficacy and tolerability of adding prescription omega-3 fatty acids $4 \mathrm{~g} / \mathrm{d}$ to simvastatin 40 $\mathrm{mg} / \mathrm{d}$ in hypertriglyceridemic patients: an 8-week, randomized, double-blind, placebocontrolled study. Clin Ther 2007;29:13541367.

23 Mohiuddin SM, Pepine CJ, Kelly MT, et al: Efficacy and safety of ABT-335 (fenofibric acid) in combination with simvastatin in patients with mixed dyslipidemia: a phase 3 , randomized, controlled study. Am Heart J 2009;157:195-203.

24 Ballantyne CM, Bays HE, Braeckman RA, et al: Icosapent Ethyl (eicosapentaenoic acid ethyl ester): effects on apolipoprotein C-III in patients from the MARINE and ANCHOR studies. J Clin Lipidol 2014;8:313-314.

25 Blair H, Dhillon S: Omega-3 carboxylic acids $\left(\right.$ Epanova $\left.^{\circledR}\right)$ : a review of its use in patients with severe hypertriglyceridemia. Am J Cardiovasc Drugs 2014;14:393-400.

26 Kastelein JJ, Maki KC, Susekov A, et al: Omega-3 free fatty acids for the treatment of severe hypertriglyceridemia: the Epanova for Lowering Very High Triglycerides (EVOLVE) trial. J Clin Lipidol 2014;8:94-106.

27 Gaudet D, Brisson D, Tremblay K, et al: Targeting APOC3 in the familial chylomicronemia syndrome. N Engl J Med 2014;371:22002206.

28 Harris WS, Ginsberg HN, Arunakul N, et al: Safety and efficacy of Omacor in severe hypertriglyceridemia. J Cardiovasc Risk 1997;4: 385-391. 